\title{
Surgical management of septic knee arthritis with open arthrotomy and debridement-a case report
}

\author{
Neetin Pralhad Mahajan, Kartik Prashant Pande*, Ravi Rameshbhai Dadhaniya, \\ Pritam Talukder
}

Department of Orthopaedics, Grant Government Medical College and Sir JJ group of Hospitals, Mumbai, Maharashtra, India

Received: 21 August 2021

Accepted: 02 October 2021

*Correspondence:

Dr. Kartik Prashant Pande,

E-mail: kartik.pande0393@gmail.com

Copyright: (C) the author(s), publisher and licensee Medip Academy. This is an open-access article distributed under the terms of the Creative Commons Attribution Non-Commercial License, which permits unrestricted non-commercial use, distribution, and reproduction in any medium, provided the original work is properly cited.

\begin{abstract}
Septic arthritis is an inflammatory destruction of the native joint following inoculation of pathogen. Most common organisms causing septic arthritis are Staphylococcus and Streptococcus. Large joints are commonly involved with hip and knee joint accounting for approximately $60 \%$ of the total cases. Diagnosis is usually straightforward with the patient presenting with obvious local signs and symptoms along with toxic constitutional symptoms owing to the aggressive nature of the disease. Medical management in form of intra-venous antibiotics forms the mainstay of treatment but it is often required for a prompt surgical intervention in order to provide acute relief from symptom and also to decrease the disease load so as to save the joint from irreversible damage. We have a 63-year-old male patient came presented to us with a right knee swelling and tenderness of 3 weeks duration with restricted ROM with severe toxic constitutional symptoms of 1 week duration. Patient was planned for open arthrotomy and debridement and drainage of the pus and was started on an empirical therapy of injection piperacillin and tazobactam combination for 3 weeks. Immediate relief from symptoms following arthrotomy with good range of motion at 4 weeks post-surgery. As is clear from our case, an early diagnosis of septic arthritis and starting of appropriate antibiotics along with appropriately aggressive surgical interventions in the form of open debridement is the key for treatment of septic arthritis in order to save the joint from irreversible inflammatory damage. Surgical intervention not only gives immediate symptomatic relief but also decreases the load over antibiotics and increases local blood supply subsequently helping in better healing.
\end{abstract}

Keywords: Septic arthritis, Arthrotomy, Debridement, Empirical therapy

\section{INTRODUCTION}

Septic arthritis is the inflammatory destruction of the native joint following inoculation of a pathogen either from haematogenous route or direct inoculation following trauma. Septic arthritis most commonly occurs due to mainly bacterial pathogens though mycobacterial, fungal, viral, and other uncommon pathogens can cause septic arthritis among the bacteria, Staphylococcus and Streptococcus accounts for nearly 90 percent of the total septic arthritis. ${ }^{1}$ Risk factors favouring pathogen inoculation and causing increased severity of the disease include rheumatoid arthritis, osteoarthritis, diabetes, prosthetic joint, intra-articular steroid injection and alcoholism to list a few. Large weight bearing joints are more commonly involved than small joints with the hip and knee joints accounting for nearly $60 \%$ of total cases. ${ }^{2}$ About $22 \%$ cases show involvement of more than one joint owing to the hematogenous route of spread. ${ }^{3}$ Despite of antibiotic use mortality rate is about $7-15$ percent in hospitalized patients. Mainstay for the treatment of septic arthritis is medical management with culture sensitive antibiotics with added surgical interventions like arthrotomy, debridement, synovectomy, and arthrodesis. Surgical intervention most often becomes necessary as the medical management with intra venous antibiotics is not 
sufficient enough to halt the disease process as the inflammatory response and the subsequent pus formation is aggressive and severe enough to destroy the joint thereby affecting the patient's daily activities morbidly disabling him along with associated severe disabling pain affecting the sleep cycle. Most often the patient presents to us when the disease has progressed significantly, pus formation is significant causing swelling of the knee joint and has started affecting his activities of daily living. If left untreated can completely destroy the joint causing sequalae such as severe arthritis, osteomyelitis of the bones involved in the joint formation, dissemination of the pathogen causing septicaemia and in around $7-10 \%$ of cases can cause mortality warranting our initial aggressive management plan. ${ }^{4}$

\section{CASE REPORT}

A 63 years old non comorbid male patient came to OPD with complaints of pain, swelling, and restriction of movement at right knee joint for 3 weeks. The swelling was non traumatic, insidious in onset and gradually progressive till it restricted his range of motion across the knee joint. It was associated with pain which was throbbing type, diffuse in nature, gradually progressive in intensity, increased on knee range of motion and relieved on rest and analgesics. It was associated with constitutional symptoms such as fever-high grade with chills and rigors, subsiding on taking oral anti-pyretics, malaise, weakness, and toxaemia like features. On examination of the knee joint-there were obvious local signs of inflammation such as local rise of temperature, redness, swelling (Figure 1) with fullness over the supra patellar pouch, diffuse tenderness. The patient had avoided going to the health facility in order to exposure to the coronavirus, the same also affecting a majority of routine medical, surgical and laboratory services making the patient apprehensive of visiting a doctor which resulted in aggravation of his symptoms over the last week making him unable to stand and walk and carry out his daily activities including those involving flexion of the knee. On preliminary imaging with $\mathrm{x}$-ray-there was no obvious traumatic bony injury to justify the severity of his pain and the only findings were peri-articular osteopenia along with joint space narrowing and osteophytes in accordance with the age of the patient (Figure 2). On further evaluation with ultrasonography, thickened synovium was seen with fluid in joint space which augmented our clinical suspicion going towards diagnosing septic arthritis. Aspiration under USG guidance was not attempted as it can augment the tract formation if mycobacterium were harbouring at joint. On haematological examination the most important of the values being ESR, CRP and WBC count were found to be raised [64, 2.4 and 20,000 in appropriate units respectively] and the patient was kept on weekly follow up of the same to monitor the disease progression/resolution. The patient was started on an empirical intra venous antibiotic combination of injection piperacillin and tazobactam and an open arthrotomy along with thorough debridement was planned to reduce disease load at affected side and to subsequently provide symptomatic relief to the patient along with reduction in the morbidity.

\section{Surgical technique}

The patient was placed in a supine position over the operating table. Induced under all aseptic precautions and under spinal and epidural anaesthesia. Scrubbing, panting and draping was done. Anterior approach for knee was employed by taking a midline incision of about $15 \mathrm{~cm}$ extending from just proximal to the superior pole of patella up to the tibial tuberosity with the knee in 90-degree flexion. Skin, subcutaneous tissue incised and flaps raised quadriceps tendon, patella and patellar tendon on both sides. A medial para-patellar incision was taken keeping 2 $\mathrm{cm}$ medial from patellar margins leaving buttress for suturing and the patella was subluxated laterally to expose the joint. After exposing the joint, exuding pus was drained and a thorough wash with adequate amount of normal saline was given intra-articularly (Figure 3), removable osteophytes were scooped out. A hypertrophied synovium along with copious number of necrotic debris (Figure 3) was visualized which was removed from the anterior, medial and lateral gutters in order to remove all the diseased tissue. Destroyed cartilage was removed. The exposed cartilage is drilled for stimulating fibrocartilage growth over cartilage defects. Extensor retinaculum is sutured properly after achieving haemostasis. Closure was done in layers over negative suction drain (Figure 4). Sterile dressings given with compression bandage. Immediate post-op the patient was stable and was shifted to the non-ICU ward. Evacuated pus, synovium and cartilage tissue were sent for culture and sensitivity examination, histopathological examination and gene xpert evaluation. Post-operative check dress was done on 3rd day. No induration was seen. The patient was kept on a skin traction over a simple pulley with $3 \mathrm{~kg}$ weight to reduce joint pain and spasm as well prevent contact of eroded cartilage. He experienced significant reduction in pain especially the night cries and swelling from post op day 3. VAS score was significantly reduced from the third day. Physiotherapy was started with active assisted and passive knee ROM exercises taught to the patient. Patient was mobilized with partial weight bearing from POD-2. Suture removal was done on POD-14, with no wound complications (Figure 5).

Surprisingly, tissue culture sensitivity showed no organism grown and gene expert was negative ruling out tuberculosis. Patient was continued on injection piperacillin\& tazobactam in appropriate adult doses as empirical antibiotic for 7 days. ESR, CRP and total WBC count monitoring was continued in the post op period with values on $3^{\text {rd }}$ day post-surgery being 54, 2.4 and 15000. On $9^{\text {th }}$ post op day, it was 40 and 1.2 positive showing a decreasing trend indicative of a subsiding infection and reduction in the subsequent inflammatory process which had ensued in the joint following the pathogen inoculation. He experienced significant reduction in pain and swelling post-operatively from day 3 . Patient was discharged at post 
op day 14 with tablet linezolid for 3 weeks and kept on weekly follow up. The patient attained flexion movement up to 90 degrees by post operative day 28 (Figure 6) with ESR, CRP and total WBC count values being 5 , negative and 10000 respectively.

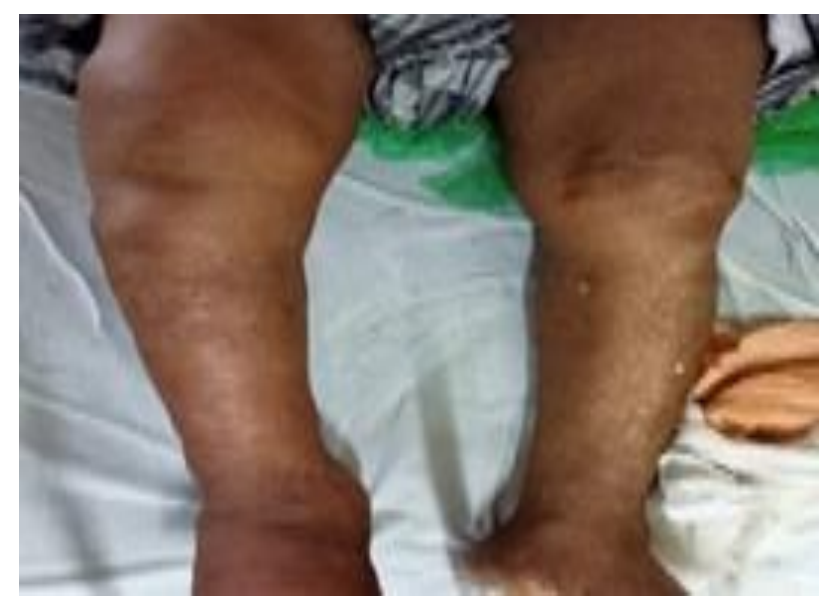

Figure 1: Clinical picture of swelling and fullness of the right knee.

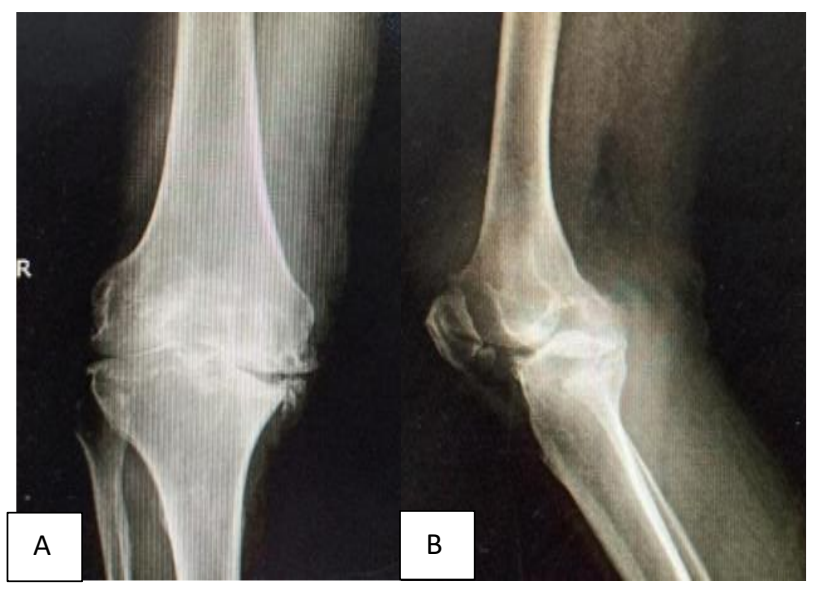

Figure 2 (A and B): X-ray of peri-articular osteopenia and arthritic changes.

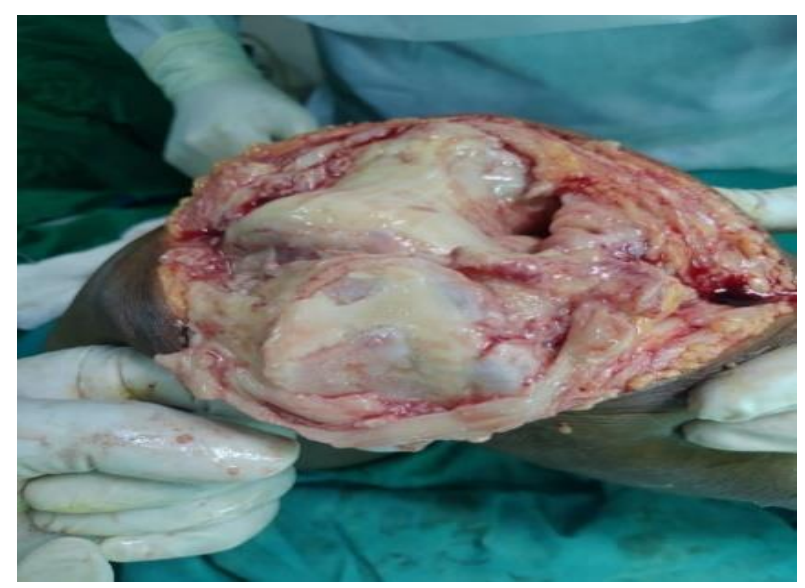

Figure 3: Knee joint of necrotic debris and hypertrophied synovium.

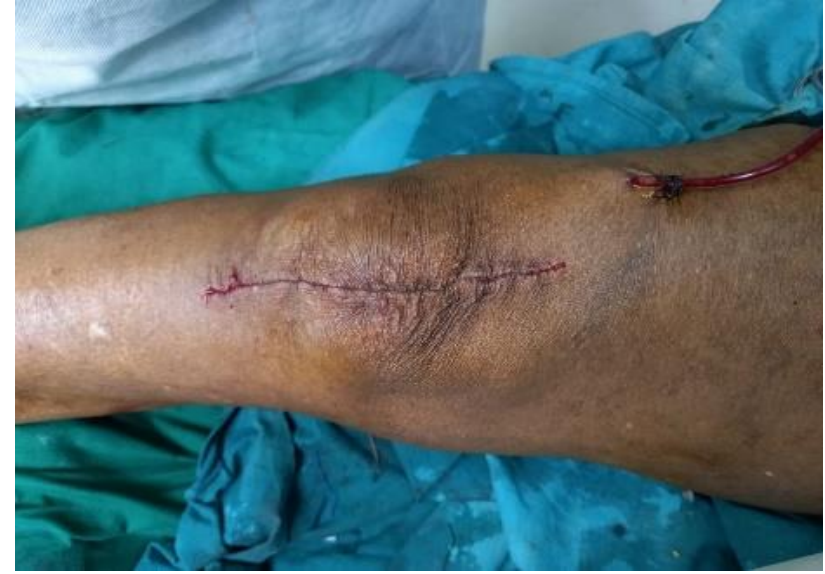

Figure 4: Closure done with negative suction drain.

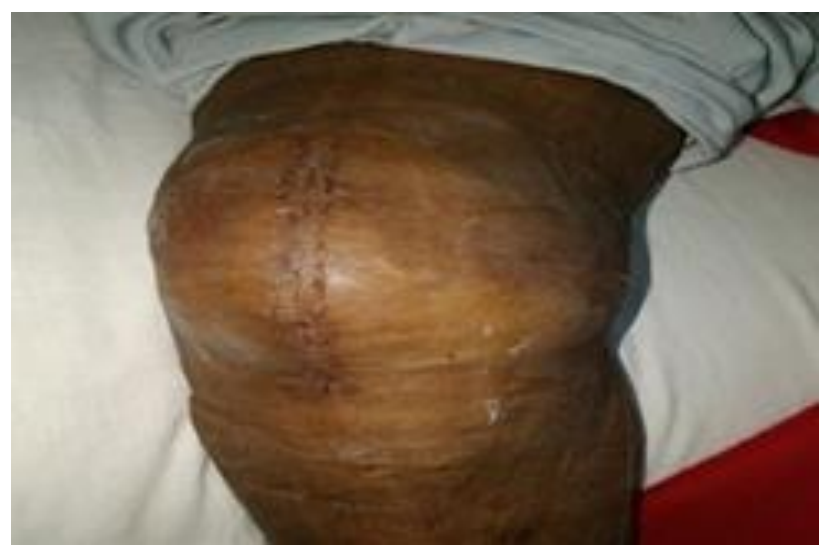

Figure 5: Suture removal at POD 14. Healthy wound.

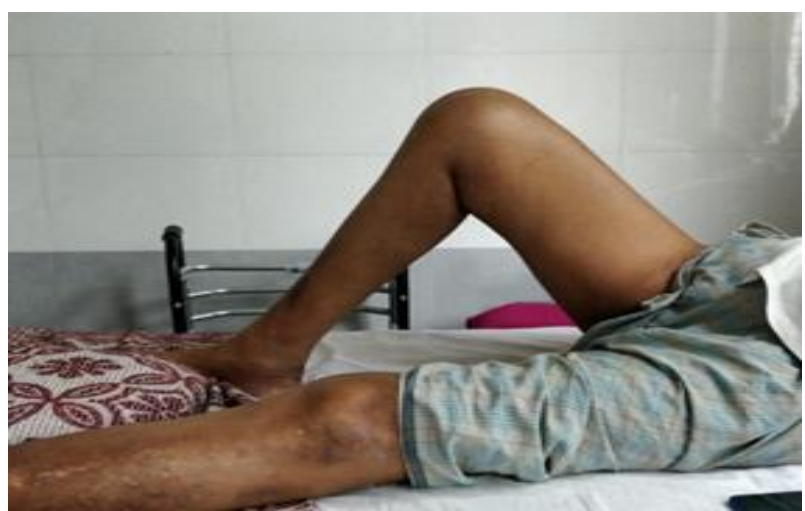

Figure 6: Knee flexion up to more than 90 degrees at POD 28 following vigorous physiotherapy with no wound complication.

\section{DISCUSSION}

Knee joint is most the common joint involved in septic arthritis as it is maximum weight bearing joint, easy for wear and tear and an easy target for pathogens. Plenty of blood supply around the joint make inflammatory process easy and so the destruction at the joint. Inflammatory cytokines play important role in disease progression. The major damage to the joint is caused by the neutrophilic 
infiltration following an aggressive inflammatory response to the pathogen, most commonly a bacterium. The neutrophilic mediated inflammatory response causes rapid effusion into the joint space causing pus formation and subsequent synovium and capsule damage cartilage destruction. ${ }^{5}$ Presumptive diagnosis can be done clinically most of the times according to Kocher's criteria for septic arthritis. X-rays are usually normal with elderly patients showing concomitant age-related arthritic changes. Other investigation in the form of ultrasonography is useful in aiding in diagnosis which will show a thickened synovium along with significant joint effusion. Lab parameters are useful adjuncts to the clinical and radiological augmentation for diagnosing in that the increased inflammatory markers pointing towards an infective source. Although the final diagnosis will be based on culture and sensitivity of the aspirated pus confirming the organism, the treatment should not be withheld until the reports are available as waiting for so long could not only delay the symptomatic relief the patient should get but also significantly destroy the joint to a point of no return as the inflammatory processes are aggressive in nature. Culture sensitivity of secretion either by USG guided aspiration or by arthrotomy is the key to find appropriate antibiotics. Blood culture plays role and shows growth in roughly half of the joint aspirate positive cases though nearly 10 percent of the cases are joint aspirate negative but blood culture positive. ${ }^{6}$ Mounting joint aspirate on blood culture is also advisable along with conventional agar plates. Medical management has certain limitations, though acting directly on pathophysiology of the disease, does not have immediate effect on symptoms. Surgical debridement, arthrotomy, synovectomy are few surgical interventions for septic arthritis. Number of debridement surgeries to be done has no clear guidelines with some results showing negative correlation. ${ }^{7}$ Arthrotomy can be done with minimal invasive modalities like arthroscopy or it can be converted to open procedure if debridement along with arthrotomy was considered as in our case where an adequate and apt arthrotomy along with drilling of the fibro cartilage as an additional procedure would help in increasing the local blood supply helping in washing out of inflammatory debris and increased antibiotic delivery to the local site for better action against the pathogen. Arthrodesis is advisable when joint reconstruction is not possible and in cases of septic arthritis following trauma and should be kept as a last resort. ${ }^{8}$ Monitoring of the septic arthritis progression can be done by weekly monitoring of ESR, CRP and total WBC count values, surgical procedure may disturb the value as in our case. Subsequent decrease in values not only shows disease progression but can also be indicators for duration of antibiotics use. The usual duration of antibiotics is 2 weeks of intra venous and 4 weeks of oral antibiotics for adequate resolution of the residual disease, if any.

\section{CONCLUSION}

As is clear from our case, an early diagnosis of septic arthritis and starting of appropriate antibiotics along with appropriately aggressive surgical interventions in the form of open debridement is the key for treatment of septic arthritis in order to save the joint from irreversible inflammatory damage. Surgical intervention not only gives immediate symptomatic relief but also decreases the load over antibiotics and increases local blood supply subsequently helping in better healing.

Funding: No funding sources

Conflict of interest: None declared

Ethical approval: No required

\section{REFERENCES}

1. Gupta MN, Sturrock RD, Field M. A prospective 2year study of 75 patients with adult-onset septic arthritis. Rheumatology (Oxford). 2001;40(1):24-30.

2. Kaandorp CJ, Dinant HJ, Van de Laar MA, Moens HJ, Prins AP, Dijkmans BA. Incidence and sources of native and prosthetic joint infection: a community based prospective survey. Ann Rheum Dis. 1997;56(8):470-5.

3. Dubost JJ, Fis I, Denis P, Lopitaux R, Soubrier M, Ristori JM et al. Polyarticular septic arthritis. Medicine (Baltimore). 1993;72(5):296-310.

4. Mathews CJ, Weston VC, Jones A, Field M, Coakley G. Bacterial septic arthritis in adults. Lancet. 2010;375(9717):846-55.

5. Hultgren $\mathrm{OH}$, Svensson L, Tarkowski A. Critical role of signalling through IL-1 receptor for development of arthritis and sepsis during Staphylococcus aureus infection. J Immunol. 2002;168:5207-12.

6. Weston VC, Jones AC, Bradbury N, Fawthrop F, Doherty M. Clinical features and outcome of septic arthritis in a single UK Health District 1982-1991. Ann Rheum Dis. 1999;58(4):214-9.

7. Peacock EE Jr. Some biochemical and biophysical aspects of joint stiffness: role of collagen synthesis as opposed to altered molecular bonding. Ann Surg. 1966;164:1-12.

8. Eralp L, Kocaoglu M, Tuncay I, Bilen FE, Samir SE. Knee arthrodesis using a unilateral external fixator for the treatment of infectious sequelae [in Turkish]. Acta Orthop Traumatol Turn. 2008;42:84-9.

Cite this article as: Mahajan NP, Pande KP, Dadhaniya RR, Talukder P. Surgical management of septic knee arthritis with open arthrotomy and debridement-a case report. Int J Res Orthop 2022;8:98-101. 